\title{
Editorial
}

\section{Call for Action in Brazil: Dealing with Misconduct in Research and Scientific Publication}

\author{
Rosemary Sadami Arai Shinkai* \\ rshinkai@pucrs.br
}

Cases of misconduct in research and scientific publications have populated the headings of both national and international news lately. There are no borders regarding incidence of misconduct in developed, developing or underdeveloped parts of the world, and all areas of knowledge are affected. This universal problem has become more evident and frequent with the increasing pressure on scholars and students to produce more results and to publish more papers in order to get academic titles and positions, job promotion, and scientific recognition in competitive research. In Brazil, it might be necessary to re-evaluate if the current procedures to assess research productivity based on quantification of publications are not creating incentives for unacceptable practices such as plagiarism, redundant publication, or guest authorship. However, the fundamental question now is how to prevent and handle misconduct cases in the scenario of productivity assessment using absolute quantitative indicators, and concurrently to promote good practices in research and publication ethics. Of course the basic action relates to continuing education of all the scientific community, particularly the formation of new investigators inside the graduate programs. However, a multiple taskforce is required as a single remedy certainly does not exist.

One recent initiative to integrate the discussion across several levels was the Second Brazilian Meeting on Research Integrity and Publication Ethics (II BRISPE) held in three different Brazilian cities (Rio de Janeiro, São Paulo, and Porto Alegre), in May 28-31 and June 1, 2012. The scope of this event was to promote a forum where researchers, editors, educators, students, and policymakers could interact and jointly learn how to face current challenges in research integrity and ethics in publication. It is still a small step towards the national discussion necessary to sensitize our entire academic and scientific community to take straightforward actions for prevention, management, and punishment of misconduct in our country.

In the macro sphere, it would be necessary to involve the integration of policies of several parties, from funding agencies to the Ministries of Education and Science, Technology and Innovation. Few Brazilian governmental institutions have already developed their own guidelines on responsible conduct of research similarly to those from several parts of the world, such as the USA and some European countries. The mechanisms to produce endorsement of a global guideline in a continental country like Brazil should be multisectorial including not only the universities and research centers, but also the regulatory and funding agencies such as CAPES, CNPq, FINEP, DECIT, and the FAPS. In 2011 one federal and one state funding agencies, the CNPq and FAPESP, respectively, released two documents to initiate the regulation of research integrity in our country. The CNPq document brings definitions of misconduct and general recommendations for researchers; overall is very broad and focus

*Dr. Shinkai graduated in Dentistry from the University of São Paulo (USP), Brazil, in 1991, and was a Research Fellow at the Department of Prosthodontics of USP from 1991 to 1996. She received her MSc (1999) and PhD (2001) degrees in Dental Clinics from the State University of Campinas (UNICAMP), Brazil, and was a Postdoctoral Research Fellow and a Visiting Professor (1999-2001, 2004) at the Department of Orthodontics of The University of Texas Health Science Center at San Antonio, USA. She is a specialist certified in Aging and Health (National School of Public Health - ENSP/FIOCRUZ, 1999), Prosthodontics (Federal Council of Dentistry, 2001), Gerontology (Brazilian Society of Geriatrics and Gerontology, 2002), and Geriatric Dentistry (Federal Council of Dentistry, 2003). She is currently Associate Professor of the Dental School of the Pontifical Catholic University of Rio Grande do Sul (PUCRS), editor-in-chief of the Revista Odonto Ciencia (Journal of Dental Science), coordinator of the Division of Research Projects at the PUCRS, Vice-President Office for Research \& Graduate Studies, Second treasurer of the Brazilian Association of Scientific Editors (ABEC) (elected for the 2012-2014 term) and Council member of the Committee on Publication Ethics (COPE) (elected for the 2012-2015 term).

J. Aerosp. Technol. Manag., São José dos Campos, Vol.4, No 2, pp. 129-130, Apr.-Jun., 2012 
on issues related to scientific writing, such as authorship and plagiarism. The FAPESP document is more comprehensive in exploring the different types of misconduct and promoting good practices, including recommendations for regulation, continuing education, and investigation/punishment mechanisms at the institutional level of institutions of higher education and research.

In relation to universities, the Brazilian Forum of Vice-Presidents for Research (FOPROP) could reach a consensus on the development and implementation of common policies to help to standardize intra- and interinstitutional processes. For example, the management of situations involving two or more institutions because of multiple authors or misconduct cases by previous employees is complex and controversial. The handling procedure would require an interinstitutional agreement or mechanisms for obtaining endorsement from all the institutions involved to reach a satisfactory resolution of the misconduct case. An umbrella regulation for universities and guidelines of responsible conduct of research also could serve as a model for the development and implementation of individual institutional policies comparable with international standards. This step is mandatory for the internationalization of our institutions of higher education and research and to establish international collaborative research projects.

In the operational aspects at the university or research center level, each institution should have a council of research integrity, and its designated president or head could be the public formal contact point to facilitate communication in cases of misconduct whistleblower and further need of internal investigation. Also, the legal department of each institution should be involved in this discussion from the early stage. Several university divisions, such as the office of graduate studies and research, office of research integrity and ethics, the university press, and the university journals should work within a common framework following a strategic plan to implement good practices and to educate the academic community and network of readers, authors, and editorial board members. Effective handling of misconduct and ethical problems in publication demands clear resolution efforts from both institutions and journals, especially in cases of fraud or plagiarism requiring not only the retraction of the publication by the journal but also simultaneous institutional action against the author.

The Brazilian journals, related institutions, and other associations (such as the Brazilian Association of Scientific Editors (ABEC) or societies of researchers, editors, administrators are other targets for action and dissemination of good practices among members, in their meetings and website. For example, ABEC is responsible for organizing two annual meetings in different Brazilian cities, aiming to give training and continuing education in scientific writing and publication matters to members and non-members all over the country. However, less than 400 Brazilian journals of all areas of knowledge are affiliated with ABEC or the BVS Virtual Community of Scientific Editors, which provide educational material and additional discussion on editorial policies. "Publication Ethics" was the theme of the ABEC meeting in 2011, because we still need to disseminate editorial good practices and guidelines in Brazil according to international standards. Currently, only a small percentage of journals published in Brazil follow explicitly international editorial guidelines such as the "Uniform Requirements for Manuscripts Submitted to Biomedical Journals" by the International Committee of Medical Journal Editors (ICMJE) or the recommendations by the Committee on Publication Ethics (COPE). This is fundamental to improve the quality of the Brazilian journals, which are also very under-represented in such international organizations and need to adopt editorial policies in synergy with the best publications in their field.

In summary, the advancement of the Brazilian scientific production with quality and responsibility involves clear definition of roles and commitment of all actors, from the Federal Government to the individual. Only with integration of policies and actions at multiple levels to qualify our research in scientific and ethical terms it will be possible to create a culture of responsible conduct of research and publication to gain international credibility and equity of conditions for collaborative research. A great challenge faces us all, but, ultimately, it is a matter of the most basic exercise of citizenship in responsible science and respect to yourself and others. 